\title{
THE ANGULAR DIAMETER OF THE SUN, AS MEASURED DURING PARTIAL PHASE OF THE ECLIPSE OF APRIL 28, 1930
}

\author{
BY
}

W. H. WRIGHT

While the solar eclipse of April 28, 1930, was observed from a point on the path of totality by a party sent from the Lick Observatory, the proximity of the path to Mount Hamilton suggested that consideration be given to the possibility of employing some of the permanently established instruments in the observation of the phenomenon. It will be recalled that the eclipse in question began and ended as an annular one, in the Pacific and the Atlantic oceans respectively, but was barely total for a short length of its path in western North America. The line of total phase passed about 90 miles, or some 150 kilometers, northwest of Mount Hamilton.

The peculiar circumstances of the eclipse suggested that a measurement be attempted of the angular diameter of the Sun, and, with the cordial approval of Director Aitken, arrangements were made to take the necessary photographs with the 36-inch refractor. There is, of course, nothing novel in such an undertaking, for the diameters of both the Sun and the Moon have been measured at time of partial eclipse on several occasions. ${ }^{1}$ There were, however, certain advantages promised by this eclipse in respect to measurements of the kind, some of which are referred to in a preliminary report ${ }^{2}$ from which the following extracts are, for convenience, taken:

"The primary object in making the photographs was the measurement of the diameter of the Sun. That a partial eclipse can be utilized for this and other purposes has long been realized, and it has been especially recognized that the measurement of the separation of the cusps may provide diameter determinations that are free from some of the systematic sources of error, for example, irradiation, that attend the more direct measurement of the limb. The measurement of the cusps is, in certain respects, equivalent to the measurement of two rather widely separated stars, an operation that is affected in only a secondary degree by irradiation and by some of the commoner instrumental and optical imperfections of astronomical telescopes." (It may be remarked that irregularities of the Moon's limb are, generally speaking, without effect on the measurements, since the point of a cusp is always on the limb of the Sun. It is only in case a cusp were actually blunted by an approximately sheer lunar cliff that disturbance would be anticipated.)

${ }^{1}$ e.g., Hayn, A. N., 201, 185, 1915; Stearns A. J., 37. 33, 1926.

${ }^{2}$ Publ. A. S. P., 42, 145, 1930.
“ . . . solar 'crescents' were photographed by the Lick Observatory party in Australia in 1922, while plans to continue the observations in 1923, at the California eclipse, were rendered ineffective by clouds. These two eclipses were of comparatively long duration and were observed from points close to the middle of the path of totality. It is worthy of note that these conditions, so desirable for most eclipse observations, are not advantageous in respect to the special measurements here referred to. If the angular diameter of the eclipsing Moon is considerably larger than that of the Sun, the arc of the exposed crescent is relatively short; thus the crescents as photographed in Australia in 1922 have a length of only about $60^{\circ}$, which renders them unsuitable to the purpose here especially considered. A little reflection shows that if the apparent diameters of the Sun and Moon are nearly equal (the condition for a short eclipse), the cusps will fall approximately at extremities of a solar diameter for a considerable interval of time in the neighborhood of the moment of conjunction, while if the observer is a little off the path of totality, the crescent will, at this time, revolve rapidly about the Sun's center and provide opportunity for measuring the diameter through a great range in position angle. These conditions were met by the recent eclipse as seen from a number of observatories in central California.

"Arrangements were accordingly made to attempt the necessary observations at the Lick Observatory. The photographs were taken with a disposition of apparatus already partly described, exposures being made by a "focal plane" shutter. In such a shutter a narrow slot is driven rapidly across in front of the photographic plate, in this way effecting an exposure of very short duration. Since it seemed desirable to have the two cusps photographed as nearly simultaneously as possible, the shutter was mounted in such a manner that it could be rotated in order to make the slot parallel to the line of cusps for each exposure. An automatic break circuit permitted a chronograph record of the instant of transit of the exposing slot over the position of the line of cusps.

"The program of observations as planned contemplated a series of 11 photographs to be made between eight minutes before and eight minutes after maximum obscuration, so spaced in time as to secure approximately equal angular intervals between succes-

VOLUME XVII 
sive cusp lines. In this way it was hoped to make diametral measures in position angles from $-21^{\circ}$ to $+123^{\circ}$, or through a total range of $144^{\circ}$. Closer proximity to the path of totality would have permitted a still greater sweep in position angle.

"As has already been stated, the carrying out of this plan was prevented by unfavorable weather. A heavy bank of fog, driven by a high wind, obscured the sky up to the time of maximum eclipse. Thereafter the Sun was more or less clearly seen at intervals, and attempts were made, by means of exposures very much longer than those anticipated, to make some record of the spectacle. Most of these photographs were either under- or overexposed, but a few were of a density suitable for measurement."

The available photographs are three in number. Inasmuch as they were made from five to seven minutes after maximum obscuration the rotation of the cusps between the first and last observations was only about $10^{\circ}$. The mean of the position angles was approximately $117^{\circ}$.

Measurement of the Plates.-The critical distance to be measured was the separation of the cusps. That distance is a little more than $16 \mathrm{~cm}$, and is beyond the capacity of any of the measuring instruments in the possession of the Lick Observatory. When this circumstance was brought to the attention of Director W. S. Adams, of the Mount Wilson Observatory, he very generously offered the use of one of the measuring engines belonging to that institution. The plates were therefore taken to Pasadena and measured on June 19, 1933. It is a pleasure to acknowledge the many courtesies extended by Dr. Adams and the members of the Mount Wilson Observatory staff on that occasion.

The engine was calibrated by measurement of the glass scale "A" belonging to the Lick Observatory, so that the distances refer to the system of that scale. ${ }^{3}$

The observations are recorded and the reductions outlined in the accompanying table. Columns (1) and (2) designate the image measured. Column (3) records the Pacific Standard Time of the observation, and (4) the measured cusp separation. Column (5) gives the calculated correction for differential refraction. In (6), under $R_{2}$, is given the value of the radius of the Sun's image at the time of measurement, derived from the quantities in the preceding two columns through allowance for the relative positions of the Sun and Moon. The dimension governing this reduction is the middle ordinate of the

${ }^{3}$ Publ. A. S. P., 45, 92, 1933.

$\begin{array}{lccccc}\text { (1) } & \begin{array}{c}(2) \\ \text { Plate }\end{array} & \text { Image } & \text { P.S.T. } & \text { Cusp separation } & \text { Ref. corr. } \\ \text { III } & 1 & 11^{\text {b }} 2^{\mathrm{m}} 40 \mathrm{~s} 92 & 16.1770 \mathrm{~cm} & +.0045 \\ \text { III } & 2 & 114 & 15.40 & 16.1590 & +.0046 \\ \text { IV } & 1 & 115 & 5.68 & 16.1694 & +.0047\end{array}$

visible limb of the Sun with respect to the chord joining the cusps; it was taken by direct measurement from the photograph and given the appropriate correction for differential refraction. (7) gives the corrections necessitated by the circumstance that the plates were taken at a temperature of 8.3 Cent. and were measured at $23{ }^{\circ}$. The quantities in column (8) are obtained by the application of these corrections, and represent the radius of the images at the time the photographs were taken. (9) gives the equivalent in seconds of are, which is the apparent angular diameter of the Sun as seen from the point of observation. Reduction to the center of the Earth, and to the mean distance of the Sun provides the entries in column (10).

A comment is necessary relative to the conversion of the linear distances $R_{1}$ in column (8) into the angular equivalents given in (9). This was effected through the relation:

$$
\tan r^{\prime}=\frac{R_{1}}{f_{w}}
$$

where $f_{w}$ is what has been referred to elsewhere as the "working focal length," or distance of the photographic plate from the second principal point of the objective. ${ }^{4}$ In these observations $f_{w}$ differs from the true focal length of the objective by about $4.6 \mathrm{~cm}$, due to the fact that the plate was that amount out of focus. The explanation of this maladjustment, from which no serious effect on the observations is believed to have resulted, is that with the objective diaphragmed down to 5 inches $(12.5 \mathrm{~cm})$, the determination of focus was difficult. ${ }^{5}$ The days preceding the eclipse were cloudy and offered but one or two fleeting opportunities for focusing, and then only on the Sun's limb, and under poor conditions of seeing. Inasmuch as the photographs were to be taken by light of the visible part of the spectrum, the attempts at adjustment were made by eye, and it was not until check measurements were made after the eclipse that the discrepancy above noted was discovered. The blurring factor due to the maladjustment is 4 ", which is undoubtedly less than the tremor disc due to bad "seeing" at the time the observations were made, while the influence on scale is completely compensated for by the use of $f_{w}$ instead of $f$ in the calculation of $r^{\prime}$. The value of $f_{w}$ used in these calculations is $17.546 \mathrm{~m}$, [ $\left.\log =1.24414\right]$.

${ }^{4}$ See this Bulletin, p. 51.

5 The error in focus, while it seems rather large, would be the equivalent of one of .02 inches in a telescope of 5 inches aperture and 5.8 feet focal length, that is to say in a telescope of the aperture here employed, and of more or less the usual focal length.

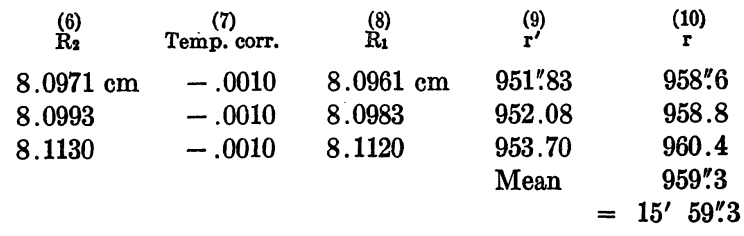


The measures of single photographs were in each case extraordinarily consistent, the range being generally less than the equivalent of 0.05 , and the rather wide scatter of the observations, as shown in column (10) at first seemed difficult to understand. It is undoubtedly to be accounted for by atmospheric disturbance during the storm conditions that prevailed at the time of the eclipse. Since the used aperture of the lens was only $12.5 \mathrm{~cm}$, the beams of light coming from the two cusps were completely separated beyond a point 15 meters in front of the telescope, and the two beams therefore passed through separate columns of air during substantially all their travel through the Earth's atmosphere. Each beam was consequently influenced by its own peculiar set of atmospheric disturbances, a circumstance which might be expected to alter momentarily the apparent separation of the cusps. This seems quite a suffcient explanation of the range in the observed values of the semidiameter.

Within limits of observational error the mean of the measures, $15^{\prime} 59^{\prime \prime} 3$, agrees with Auwers's value, 15' 59.6 , resulting from his discussion of many series, or groups, of heliometer observations. ${ }^{6}$ It may be worth noting that the range in the single measures in column (10) is but little in excess of that among the several groups of observations used by Auwers (extreme values; Hartwig, Strasbourg, 1883, 960"35; Löw, Mauritius, 1874?, 958".80).

One of the purposes in undertaking these measurements was to apply a test for the presence, close to the Moon's shadow cone, of unusual refractive effects. In relation to the apparent displacement of stars seen near the eclipsed Sun-the so-called relativity displacement - the question has been raised as to the possible presence, in the neighborhood of the Moon's shadow, of a refractive disturbance, symmetrical with respect to the eclipse axis, due to local cooling of the air. It was recognized by Professor C. L. Poor that such a disturbance would be expected to influence not only the apparent positions of the stars, but the apparent diameters of the Sun and Moon as well. He suggested that, in order to

${ }^{\circ} A . N ., 128,367,1891$. test the matter, observations of these diameters be made under eclipse conditions. Acting upon this suggestion, Miller and Marriott measured the diameter of the Moon as seen against the corona at the eclipse of January 14, 1926. ${ }^{7}$ They found the diameter to be normal. While there was no reason to question the validity of their result, which was most carefully derived, it seemed worth while to take advantage of the very favorable opportunity to measure the diameter of the nearly eclipsed Sun, provided by the event of 1930 .

In the degree to which it is permissible to attach significance to so limited a number of measurements as that available in this inquiry, it is to be inferred that the Sun, as observed under conditions of nearly total eclipse on April 28, 1930, maintained its normal apparent angular diameter. Rather than indicating abnormality, the observations suggest that the procedure adopted in securing them might, under favorable conditions of observation, prove useful in providing an independent measurement of the true angular diameter of the Sun. It is to be remarked that in one particular the 1930 eclipse may not be regarded as representative of eclipses at which the stellar spreading has been observed; the shadow cone, on that occasion, was relatively small. It would be difficult to estimate the relation of the size of the shadow to the amount of refraction to be expected within it, but, inasmuch as the critical rays traverse only a part of the cone, any such refraction would depend upon the temperature gradient, rather than upon the total drop in temperature in the neighborhood of the eclipse path, and there appears to be no reason to assume that this would be markedly less for a small than for a large shadow. In any event, the absence of any evidence whatever of abnormal refraction in the vicinity of the axis of the 1930 eclipse, corroborative as it is of the similar negative finding by Miller and Marriott in respect to the diameter of the Moon at the eclipse of 1926, is discouraging to the notion that the observed displacements of stars viewed near the eclipsed Sun are explicable in terms of disturbed atmospheric refraction within the Moon's shadow.

Lick Observatory

October, 1934,

Issued November 10, 1934 